\title{
Design of Stable Controller for Flexible Solar Panel by $\mathbf{H}_{\infty}$ Loop-Shaping Method
}

\author{
Dini Wang $\mathbb{D}$, ${ }^{1}$ Fanwei Meng $\mathbb{D}{ }^{1}$, Shengya Meng, ${ }^{1}$ and Aiping Pang $\mathbb{D}^{2}$ \\ ${ }^{1}$ School of Control Engineering, Northeastern University at Qinhuangdao, Qinhuangdao 066004, China \\ ${ }^{2}$ College of Electrical Engineering, Guizhou University, Guiyang 550025, China
}

Correspondence should be addressed to Fanwei Meng; mengfanwei@neuq.edu.cn

Received 2 May 2020; Revised 22 May 2020; Accepted 2 June 2020; Published 28 June 2020

Guest Editor: Shubo Wang

Copyright (c) 2020 Dini Wang et al. This is an open access article distributed under the Creative Commons Attribution License, which permits unrestricted use, distribution, and reproduction in any medium, provided the original work is properly cited.

Flexible solar panels play an essential role in the field of aerospace. However, many difficulties appear in the control design due to the existence of a weakly damped resonance module. The design for flexible systems often causes an unstable controller so that the systems after design still have trouble in putting into practice. We adopt $\mathrm{H}_{\infty}$ loop-shaping design and put forward a directive method for selecting the weighting function. The simulation results indicate that system bandwidth is optimized based on the stable controller. In this way, the control precision and response speed of the system are improved. In the meantime, the system is easy to put into use.

\section{Introduction}

With the rapid development of aerospace, most spacecraft absorb solar energy to meet their energy needs [1]. One end of the solar array is connected to the satellite, and the other end is free to extend. Such structures are called solar panels [2]. When the satellite is launched, the solar panel is folding; after the spacecraft gets into orbit, the solar panel is expanded. So, the solar panel often belongs to a multibody structure, and the mass is light. The larger the spacecraft is, the more energy it requires so that its area will be larger and its structure will be more complicated. For the typical flexible structure on spacecraft [3], people have increasingly high requirements for its function, reliability, service life, and attitude control $[4,5]$.

Components with flexible structures have gained in popularity because of their strong reliability, good adaptability, and light weight. However, weakly damped resonance modes in the system bring many difficulties to control design. For example, they will increase the system setting time and even cause instability, which may lead to mode overflow in high-order resonant modes [6]. Many scholars are committed to solving the control design problem of such systems so as to suppress the influence of these dynamic characteristics. The analysis shows that there are unstable controllers introduced by many design methods for weakly damped flexible systems, which make the designed systems difficult to use [7]. Then, we adopt $\mathrm{H}_{\infty}$ loop-shaping design proposed by McFarlane, and we hope to optimize the system performance (bandwidth) based on the stable controller. $\mathrm{H}_{\infty}$ control design $[8,9]$ mainly involves structure problems and weighting function selection. Structural problems include the two-block problem, the four-block problem, and $\mu$ synthesis. However, no matter what the structure is, the unstable $\mathrm{H}_{\infty}$ controller may be introduced for the weakly damped flexible system [7, 10-12]. More importantly, it is difficult to improve the system performance by $\mathrm{H}_{\infty}$ loopshaping design in practice. For example, the hard disk drive in [13] shows that the system bandwidth did not increase after the design, and the system performance did not improve significantly.

The main indexes to measure the system performance are frequency bandwidth and precision [14-16]. And the frequency bandwidth, determined by the frequency response, can reflect the rapidity of the system. The larger the bandwidth is, the better the rapidity is, which not only stabilizes the flexible system as soon as possible after rapid maneuver but also reduces fatigue damage. However, the 
increase of the system bandwidth means more weakly damped resonance modes will be included so that the stability of the system cannot be ensured. Therefore, it is extremely significant to achieve an optimal state in control design, that is, to find a better trade-off between robustness and system bandwidth. How to decay rapidly and smoothly, that is, whether the system performance (bandwidth) can be improved on the basis of stabilizing the controller, is still an issue worthy of academic research.

\section{Solar Panel System Model}

The solar panel attitude control is taken as an example to design the control system in the classic literature [10] of $\mathrm{H}_{\infty}$ loop-shaping method. Here, we further analyze the design problem of flexible systems based on the system model. When only the rigid mode and the first-order mode are considered, the state-space equations of the system can be written as $[10,12]$

$$
\begin{aligned}
& \dot{\mathbf{x}}=\left[\begin{array}{cccc}
0 & 1 & 0 & 0 \\
0 & 0 & 0 & 0 \\
0 & 0 & 0 & 1 \\
0 & 0 & -\omega_{1}^{2} & -2 \zeta_{1} \omega_{1}
\end{array}\right] \mathbf{x}+\left[\begin{array}{c}
0 \\
1.7319 \times 10^{-5} \\
0 \\
3.7859 \times 10^{-4}
\end{array}\right] \mathbf{u}, \\
& \mathbf{y}=\left[\begin{array}{llll}
1 & 0 & 1 & 0
\end{array}\right] \mathbf{x},
\end{aligned}
$$

where $\omega_{1}=1.539 \mathrm{rad} / \mathrm{s}$ and $\zeta_{1}=0.003$. In (1), $\mathbf{u}$ is the control torque $(\mathrm{N} \cdot \mathrm{m})$ and $\mathbf{y}$ is the roll angle $(\mathrm{rad})$ which can be measured. The corresponding transfer function of (1) is

$$
G(s)=\frac{3.9591 \times 10^{-4}\left(s^{2}+0.0004 s+0.1036\right)}{s^{2}\left(s^{2}+0.009 s+2.369\right)} .
$$

Equation (2) can be rewritten as

$$
G(s)=\frac{1.7319 \times 10^{-5}}{s^{2}}+\frac{3.7859 \times 10^{-4}}{s^{2}+2 \zeta_{1} \omega_{1} s+\omega_{1}^{2}} .
$$

It is obvious that the amplitude of the first-order mode $c_{1}=3.7859 \times 10^{-4}$ is larger, which is an order of magnitude different from the amplitude of the rigid mode $c_{0}=1.7319 \times 10^{-5}$. So, the solar panel system has strong flexibility.

Equation (2) shows that the gain of the controlled plant is low. In the control design, we usually increase the gain of the system to suppress the possible disturbance. Meanwhile, the bandwidth should not exceed the frequency range of the first-order mode. Therefore, multiply (2) by the gain $K=$ 10000 to get $G_{1}(s)$ (see equation (4)). The corresponding Bode plot is shown in Figure 1:

$$
G_{1}(s)=\frac{3.9591\left(s^{2}+0.0004 s+0.1036\right)}{s^{2}\left(s^{2}+0.009 s+2.369\right)} .
$$

Equation (3) and Figure 1 show us that the rigid body mode component is low, which means that, in the lowfrequency stage, the characteristic of the system will shift to the first-order flexible mode after $\omega$ exceeds $0.332 \mathrm{rad} / \mathrm{s}$. In other words, it is required to ensure the system stability in the frequency stage where the amplitude-frequency characteristic drops sharply in the design. This is the difficulty of

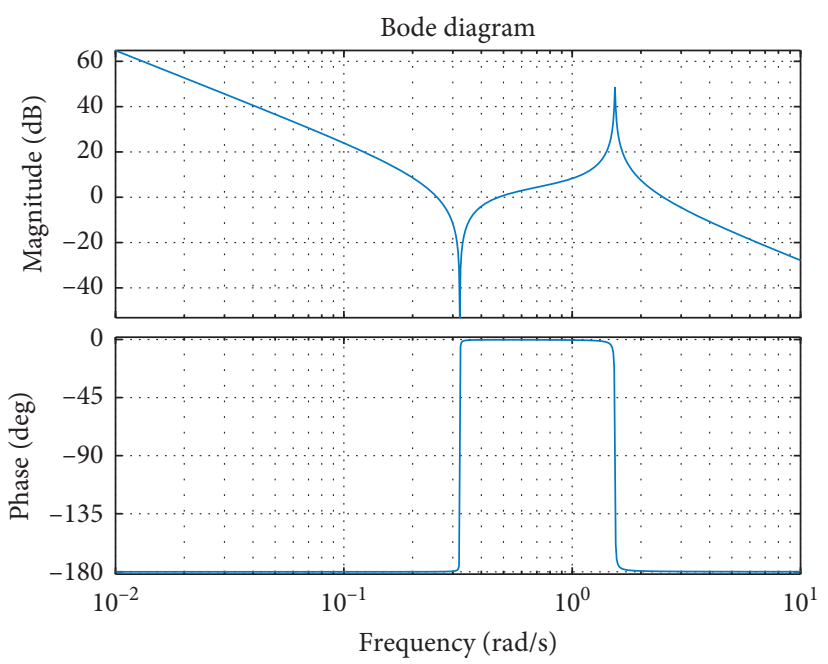

Figure 1: Bode plot of the system (4).

the weakly damped flexible system design [12]. Generally speaking, $\mathrm{H}_{\infty}$ control theory is applicable to all flexible systems. However, $\mathrm{H}_{\infty}$ loop-shaping method in $\mathrm{H}_{\infty}$ control theory is more suitable for the design of the system with weak damping. This is because $\mathrm{H}_{\infty}$ loop-shaping method just deals with the stability of the frequency stage crossing $0 \mathrm{~dB}$ line in the open-loop characteristic.

\section{3. $\mathrm{H}_{\infty}$ Loop-Shaping Design}

The basic principle of $\mathrm{H}_{\infty}$ loop-shaping design is to construct the open-loop transfer function to meet the performance requirements of the closed-loop system. The openloop transfer function here is technically referred to as "loop transfer function." According to this principle, the weighting function in the compensation link should be determined firstly according to the performance requirements (steadystate performance, dynamic performance, noise suppression performance, etc.), and then the $\mathrm{H}_{\infty}$ controller should be designed so that the system has sufficient robustness.

For the MIMO system, the transfer function matrix multiplication is divided into premultiplication and postmultiplication. So, compensation links in series are also divided into lead compensation $\left(W_{1}\right)$ and lag compensation $\left(W_{2}\right)$. The compensated plant is technically referred to as "shaped plant" $\left(G_{S}\right)$ :

$$
G_{S}=W_{2} G W_{1} .
$$

Next, the $\mathrm{H}_{\infty}$ controller $K_{\infty}$ is designed for $G_{S}$ to stabilize the shaping design system. $K_{\infty}$ and compensation links in $G_{S}$ constitute the final controller:

$$
K=W_{1} K_{\infty} W_{2} .
$$

This design method is referred to as " $\mathrm{H}_{\infty}$ loop-shaping design method," and its system structure diagram is shown in Figure 2.

The shaped plant in $\mathrm{H}_{\infty}$ loop-shaping design is described by coprime decomposition. Here, we adopt the right coprime decomposition, and then the shaped plant can be expressed as 


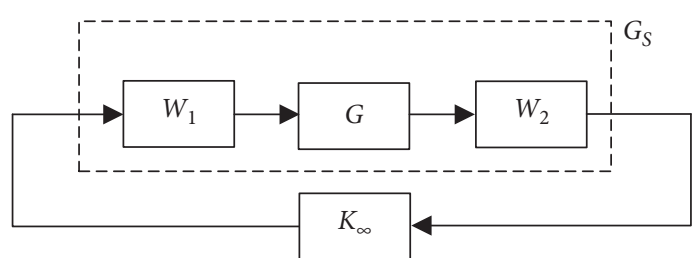

(a)

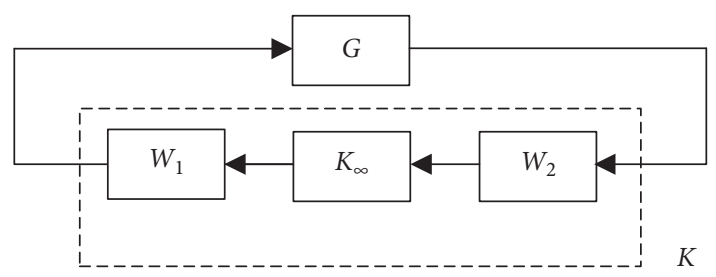

(b)

FIgURE 2: Basic system structure diagram of $\mathrm{H}_{\infty}$ loop-shaping design.

$$
G_{S}=N M^{-1}, \quad N, M \in R H_{\infty} .
$$

Since it is required to adopt normalized coprime factorization, so $N$ and $M$ in (6) should satisfy

$$
[N(-s)]^{\mathrm{T}} N(s)+[M(-s)]^{\mathrm{T}} M(s)=I .
$$

Equation (8) is equivalent to $\left[\begin{array}{ll}N & M\end{array}\right]^{\mathrm{T}}$ being an internal matrix [17].

When we use coprime factors to express the shaped plant, the perturbed shaped plant can be represented by the additive coprime factor perturbation:

$$
G_{S \Delta}=\left(N+\Delta_{N}\right)\left(M+\Delta_{M}\right)^{-1}, \quad \Delta_{N}, \Delta_{M} \in H_{\infty},
$$

where $\Delta_{N}$ and $\Delta_{M}$ are stable transfer functions. The uncertainty system of this plant is one-input two-output, and the corresponding matrix is

$$
\Delta=\left[\begin{array}{ll}
\Delta_{N} & \Delta_{M}
\end{array}\right]^{\mathrm{T}} .
$$

From the perspective of robustness, the uncertainty $\Delta$ of coprime factors is listed separately, and Figure 3 is arranged into the structure diagram as Figure 4.

$F_{l}\left(P, K_{\infty}\right)$ represents the transfer function matrix of the nominal system, where $P$ is the generalized plant constituted by $G_{S \Delta}$ except $\left(\Delta_{N}, \Delta_{M}\right)$. When using $\mathrm{H}_{\infty}$ loop-shaping design, we can obtain

$$
\min _{K}\left\|F_{l}\left(P, K_{\infty}\right)\right\|_{\infty}=\gamma
$$

Here, the stability margin $\varepsilon=\gamma^{-1}$ is generally used to reflect the system's robustness.

According to the small gain theorem, when the norm of the uncertainty of coprime factors is less than $\gamma^{-1}$, that is,

$$
\|\Delta\|_{\infty}:=\left\|\begin{array}{c}
\Delta_{N} \\
\Delta_{M}
\end{array}\right\|_{\infty}<\gamma^{-1}:=\varepsilon,
$$

the system is stable. We generally require that $\gamma$ should not be larger than $4 \sim 5[10,18]$, so that this system is still stable when the perturbation reaches 0.2 . It means that this system has sufficient robustness.

Since $\left[\begin{array}{ll}N & M\end{array}\right]^{\mathrm{T}}$ is an internal matrix, the $\mathrm{H}_{\infty}$ norm of the transfer function matrix in the nominal system is constant after left-multiplied by an internal matrix; that is,

$$
\left\|F_{l}\left(P, K_{\infty}\right)\right\|_{\infty}=\left\|\left[\begin{array}{l}
N \\
M
\end{array}\right] F_{l}\left(P, K_{\infty}\right)\right\| .
$$

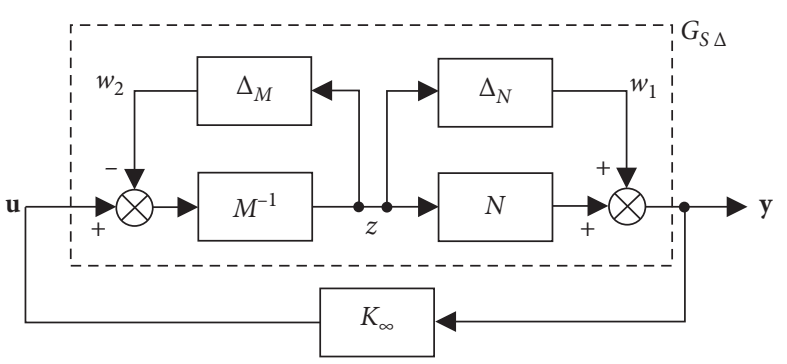

FIGURE 3: Structure diagram of the uncertainty of coprime factors.

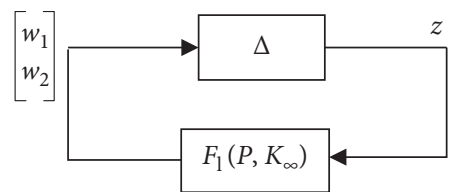

FIGURE 4: System structure diagram based on robustness analysis.

Therefore, in the $\mathrm{H}_{\infty}$ problem, the output $z$ can be rightmultiplied by matrices $N$ and $M$ to become two outputs $\left[\begin{array}{ll}z_{1} & z_{2}\end{array}\right]^{\mathrm{T}}$, as shown in Figure $5 . \mathrm{H}_{\infty}$ norm of this two-input two-output system is $\left\|F_{l}\left(P, K_{\infty}\right)\right\|_{\infty}$. The intermediate variable $z$ is no longer needed in the system shown in Figure 5, and the shaped plant $G_{S}$ can be directly used to solve the $\mathrm{H}_{\infty}$ problem. Therefore, although the loop-shaping method is an idea based on coprime decomposition, coprime decomposition is usually unnecessary in actual design [19].

The selection of the weighting function is the core problem in the $\mathrm{H}_{\infty}$ design method. The design methods in $\mathrm{H}_{\infty}$ theory, such as state feedback, output feedback, and loop-shaping, involve the selection of weighting function (or weighting coefficients) [9]. In $\mathrm{H}_{\infty}$ design, the disturbance suppression and robustness correspond to the characteristics of the low- and high-frequency stages of the system, respectively. Hence, the weighting function often relies on the requirements of low- and high-frequency stages instead of the middle-frequency stage. In fact, the middle-frequency stage of a weakly damped flexible system contains several weakly damped resonant modes that are difficult to control. Therefore, this program is different from the conventional design idea in the selection of the weighting function based on trial and error (only considering Single-Input SingleOutput systems): 


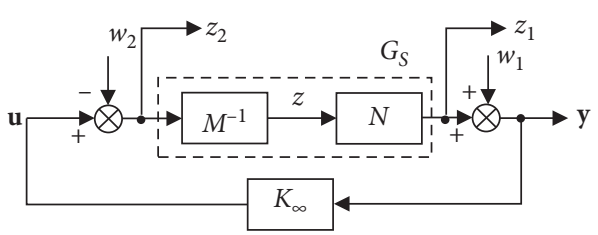

FIGURE 5: Structure diagram of a two-input two-output system.

$$
W(s)=\rho \frac{\left(s+r_{1}\right)^{a}}{\left(s+r_{2}\right)^{a}} .
$$

In (14), $r_{2}=\sqrt{\omega_{1}^{2} / k}$ and $r_{1}=k r_{2}$. follows:

The specific steps of the $\mathrm{H}_{\infty}$ loop forming design are as

Step 1. Determine the values of $r_{1}$ and $r_{2}$ by $k$.

The values of $r_{1}$ and $r_{2}$ determine the exact position of the inflection point in the frequency characteristic of the weighting function. $r_{2}$ should satisfy $r_{2} \in\left(\omega_{0}, \omega_{1}\right)$; $r_{1}$ should satisfy $r_{1} \in\left(\omega_{1}, \omega_{1}+\sigma\right)$ and $\sigma>0$, where the value of $\sigma$ should be as low as possible (if $\sigma$ is too large, it will cause the influence on unmodeled dynamics on the high-frequency stage). Therefore, the requirements of $r_{1}$ and $r_{2}$ should be considered eclectically according to the specific transfer function of the controlled object, and then the value of the parameter $k$ is selected.

Step 2. Determine the power $a$ of the pole-zero.

On the one hand, the value of $a$ determines the attenuation rate of the phase-frequency characteristic curve of the weighting function; on the other hand, it is proportional to the gain of the controller in the lowfrequency stage. Meanwhile, the values of $a$ and $\rho$ also affect the bandwidth and sensitivity of the designed system. The gain of the controller reflects the design requirement, that is, increase the gain of the system as much as possible to suppress interference. However, for flexible systems, the gain of the controller decays rapidly after first-order modal frequency, so as not to excite the high-order resonant mode in the system. So, the value of $a$ should be integrated with requirements from multiple aspects.

Step 3. Determine the value of the parameter $\rho$.

The value of $\rho$ is proportional to the gain of the controller. At the low-frequency stage, the gain of the controller corresponds to $(10)^{a} \rho$; at the high-frequency stage, the gain of the controller relies only on the value of $\rho$. According to the expected gain of the controller and the performance of the designed system such as bandwidth and sensitivity, the values of $a$ and $\rho$ should make adjustments until the designed system reaches the ideal state.

Step 4. According to the requirement of robustness, the weighting function should make alternative adjustments.

Generally speaking, the reference criterion is to make the value of $\gamma$ not larger than $4 \sim 5$. If the criterion is not met, the weighting function needs to make further adjustments. And we also can adjust the weighting function with the value of $\gamma$ not larger than $4 \sim 5$ as the restriction condition.

Step 5. Design $\mathrm{H}_{\infty}$ controller $K_{\infty}$ for $G_{S}$.

We usually use ncfsyn function in the Robust Control Toolbox of Matlab to design the controller $K_{\infty}$. Compared with the transfer function, the controller $K_{\infty}$ is added to the open-loop transfer function after system design, which mainly corrects the characteristics of the middle- and high-frequency stages to ensure the stability margin of the system and has little influence on the low-frequency stage [5]. This also indicates that $\mathrm{H}_{\infty}$ loop-shaping method not only guarantees the stability margin of the system but also guarantees the system performance and robust stability of unmodeled dynamics.

Step 6. Adjust the weighting function selectively according to the stability of the controller $K$.

If the controller $K$ obtained by the weighting function is unstable, we need to return "Step 1" to make appropriate adjustments to the weighting function.

\section{4. $H_{\infty}$ Loop-Shaping Design for the Solar Panel System}

We take the solar panel system model into the specific steps of $\mathrm{H}_{\infty}$ loop-shaping design in Section 3, so as to further analyze the system performance after design and the stability of the controller.

Step 1. Determine the values of $r_{1}$ and $r_{2}$ by $k$.

Since the first-order mode $\omega_{1}=1.539 \mathrm{rad} / \mathrm{s}$ is known, we just need to determine the values of $r_{1}$ and $r_{2}$ by $k$. The first inflection point in the amplitude-frequency characteristic of the weighted function should be between zero 0.3219 and the first-order mode $\omega_{1}=1.539 \mathrm{rad} / \mathrm{s}$. The second inflection point should be after $\omega_{1}=1.539 \mathrm{rad} / \mathrm{s}$ and not exceed too much. Otherwise, it will cause an influence on unmodeled dynamics on the high-frequency stage. Taking the above two factors into consideration, we take $k=10$ to get $r_{1}=4.876$ and $r_{2}=0.4876$.

Step 2. Determine the power $a$ of the pole-zero.

Since the value of $a$ should be integrated with the requirements from multiple aspects, we take $a$ as 5 preliminarily based on trial and error with the combination of $a$ and $\rho$.

Step 3. Determine the value of the parameter $\rho$.

$(10)^{a} \rho$ corresponds to the reciprocal of the controller gain, reflecting the requirements of system design, that is, to maximize the gain of the system to suppress interference. However, the gain of the controller decays rapidly after the first-order modal frequency, so as not to excite the high-order resonant mode in the system. Here, we take $a=5$ and $\rho=0.023$ to get $(10)^{a} \rho=2300$. 

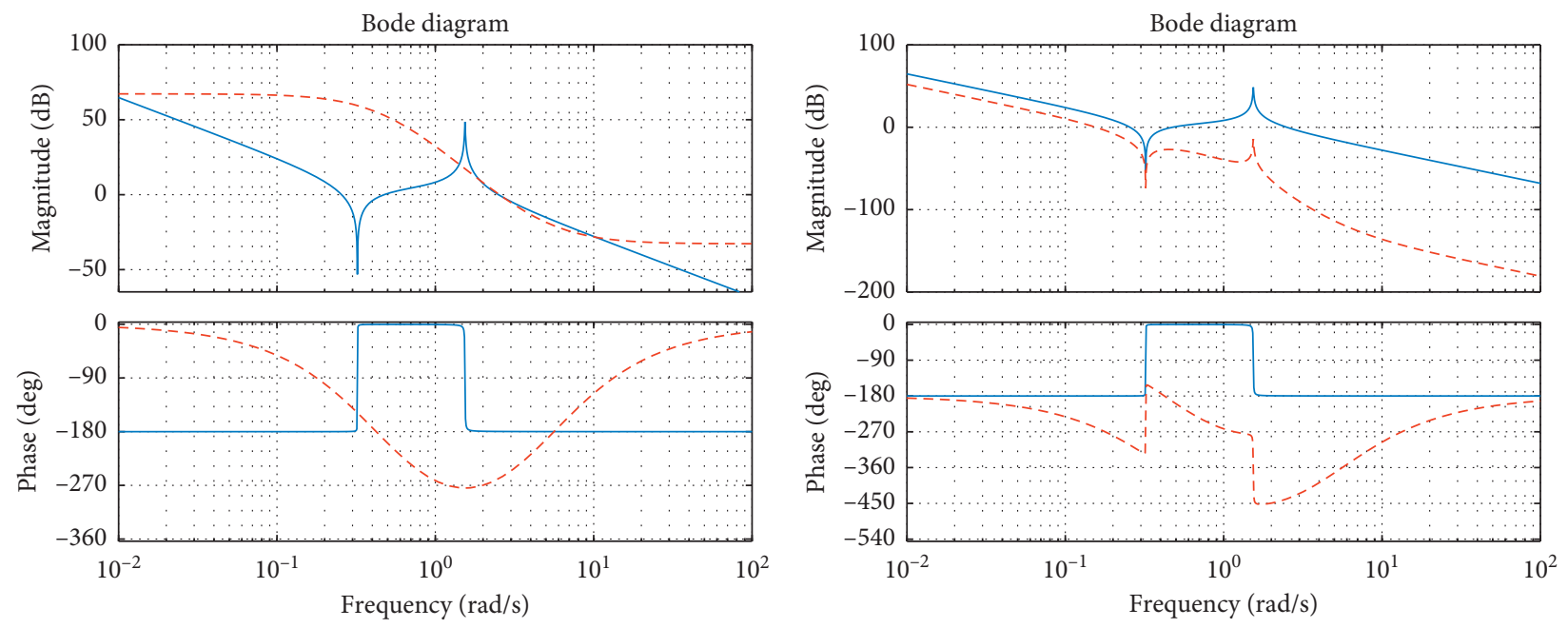

$$
-G_{1}
$$$$
-G_{1}
$$

(a)

(b)

Figure 6: Bode plot based on comparing $G_{1}(s)$ with $W(s)$ and $G_{S}(s)$. (a) $G_{1}(s)$ and $W(s)$; (b) $G_{1}(s)$ and $G_{S}(s)$.

Step 4. According to the requirement of robustness, the weighting function should make alternative adjustments.

Based on the previous three steps, the weighting function has been preliminarily determined as

$$
W(s)=0.023 \frac{(s+4.876)^{5}}{(s+0.4876)^{5}} .
$$

$$
\gamma=\left\|F_{l}\left(G, K_{\infty}\right)\right\|_{\infty}=4.7461<5
$$

This satisfies the requirement of robust stability in loopshaping design, and then we obtain the system stability margin $\varepsilon=0.2107$.

The transfer function of the shaped plant can be obtained from equations (2) and (15):

$\mathrm{H}_{\infty}$ norm of the system is

$$
G_{S}=W(s) G(s)=\frac{9.1059 \times 10^{-6}(s+4.876)^{5}\left(s^{2}+0.0004 s+0.1036\right)}{s^{2}(s+0.4876)^{5}\left(s^{2}+0.009 s+2.369\right)} .
$$

The results of comparing the amplitude-frequency characteristics of $G_{1}(s)$ with those of $W(s)$ and $G_{S}(s)$ are shown in Figure 6. We can simply conclude from the figure that the weighting function in loop-shaping design not only corrects the high- and low-frequency stages but also changes the characteristics of the middle-frequency stage.
Step 5. Design $\mathrm{H}_{\infty}$ controller $K_{\infty}$ for $G_{S}$.

We use ncfsyn function in Matlab to obtain the controller directly. After omitting the high-frequency mode, the controller is obtained as

$$
\begin{aligned}
K(s)= & \frac{0.10671(s+4.876)^{5}(s+0.3641)(s+0.06995)\left(s^{2}+1.123 s+0.3173\right)}{(s+0.4876)^{5}\left(s^{2}+1.793 s+0.8568\right)\left(s^{2}+0.1399 s+0.0928\right)} \\
& * \frac{\left(s^{2}+0.9456 s+0.2331\right)\left(s^{2}+0.00923 s+2.369\right)}{\left(s^{2}+0.9383 s+0.5095\right)\left(s^{2}+0.0009546 s+2.371\right)} .
\end{aligned}
$$




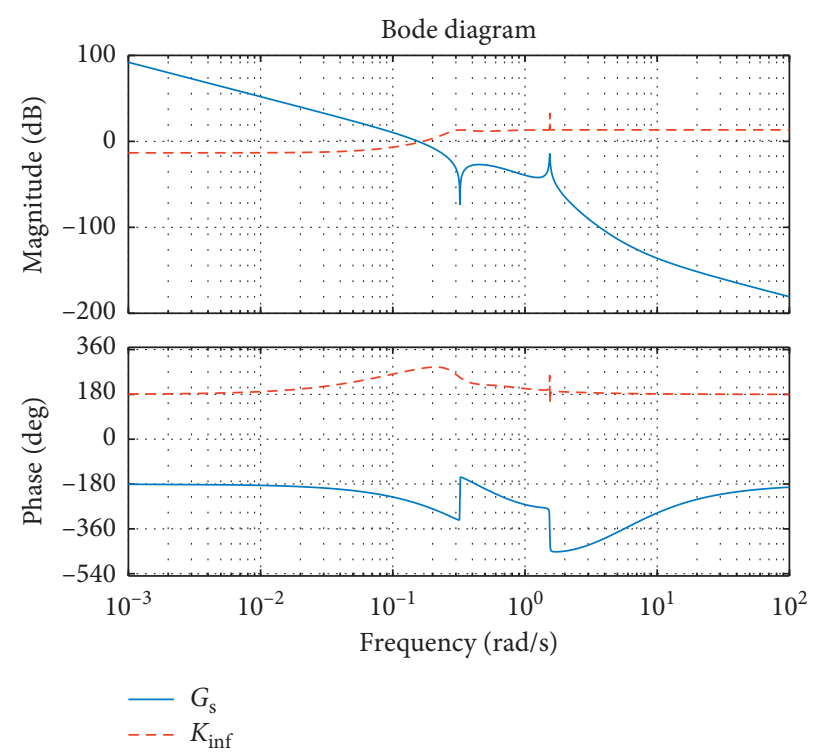

(a)
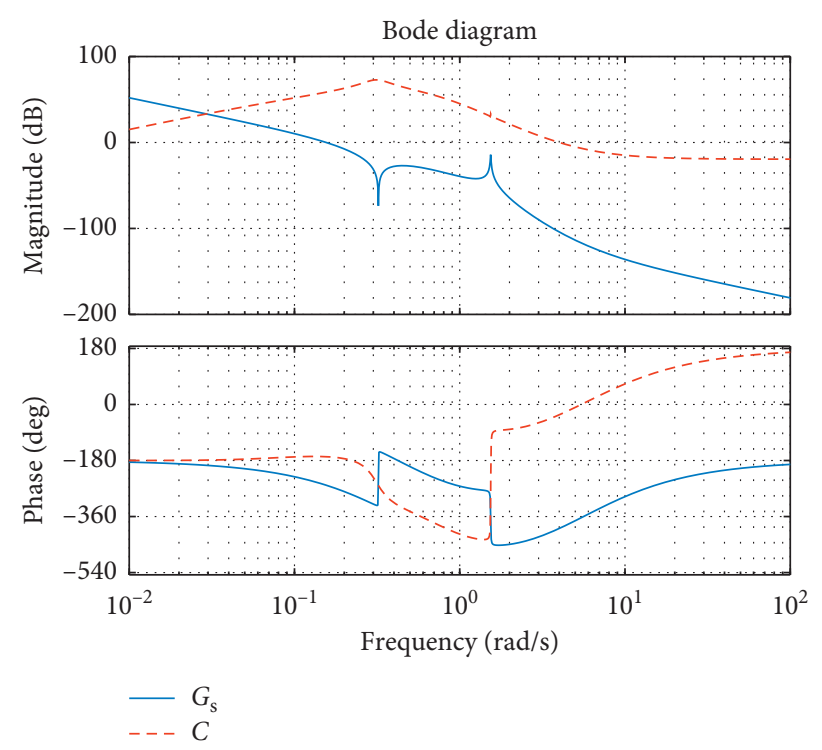

(b)

FIGURE 7: Bode plot based on comparing $G_{S}(s)$ with $K_{\infty}$ and the transfer function. (a) $G_{s}(s)$ and $K_{\infty}$, (b) $G_{s}(S)$ and the transfer function.

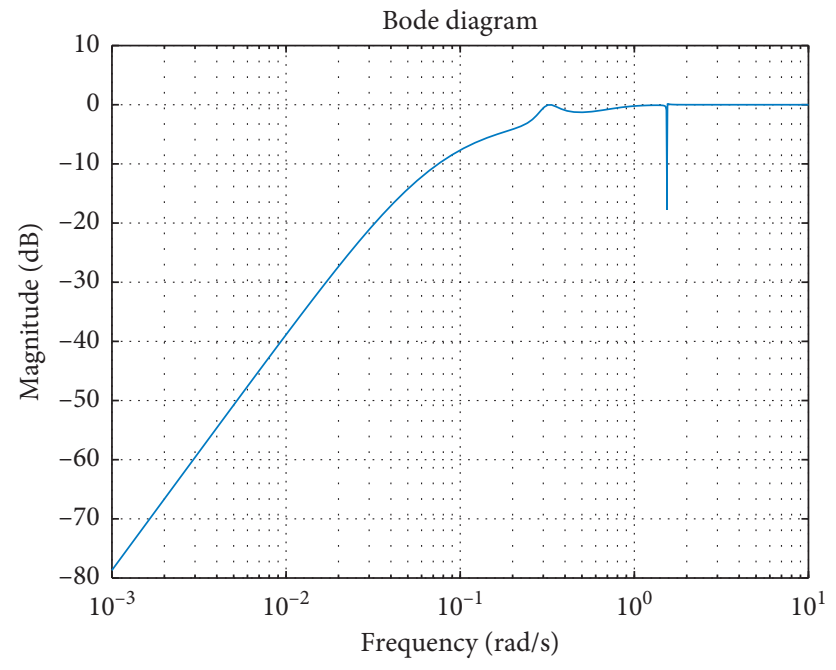

FIGURE 8: Sensitivity characteristic curve.

The corresponding $\mathrm{H}_{\infty}$ controller is

$K_{\infty}(s)=\frac{4.6396(s+0.3641)(s+0.06995)\left(s^{2}+1.123 s+0.3173\right)\left(s^{2}+0.00923 s+2.369\right)\left(s^{2}+0.9456 s+0.2331\right)}{\left(s^{2}+1.793 s+0.8568\right)\left(s^{2}+0.1399 s+0.0928\right)\left(s^{2}+0.9383 s+0.5095\right)\left(s^{2}+0.0009546 s+2.371\right)}$.

The results of comparing the amplitude-frequency characteristics of the shaped plant $G_{S}(s)$ with $K_{\infty}$ and the transfer function of the system after design are shown in Figure 7. We can simply conclude from the figure that the $\mathrm{H}_{\infty}$ controller designed by the loopshaping method makes corrections to the original $W(s) G(s)$, mainly in the middle-frequency stage to ensure stability and robustness, while only the gain is adjusted slightly on the low- and high-frequency stages.

Step 6. Adjust the weighting function selectively according to the stability of the controller $K$.

According to equation (18), the controller ${ }_{K}$ after the loop-shaping design is stable, since there is no polezero in the right half of the complex plane. 


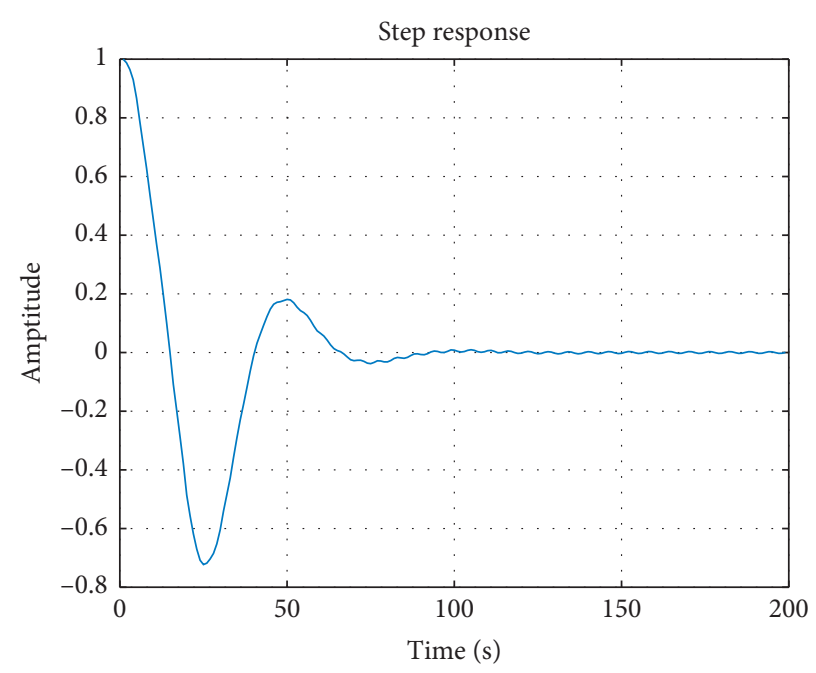

(a)

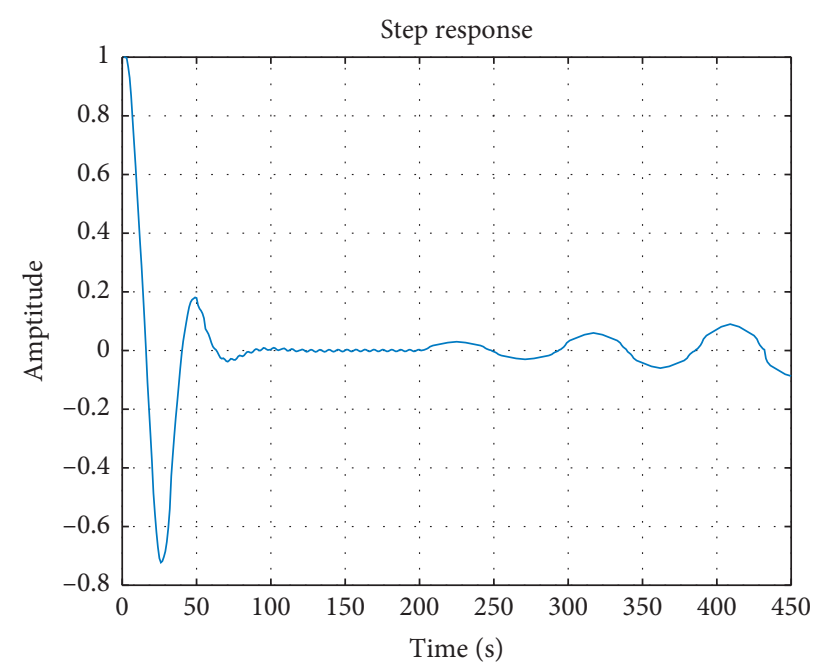

(b)

FIgURE 9: System response under step disturbance with/without perturbation. (a) The response curve of the nominal system. (b) The response curve of the system with perturbation.

The sensitivity characteristic $S(j \omega)$ of the system after the loop-shaping design is shown in Figure 8.

Figure 9 shows the response curves of the feedback control system with/without parameter perturbation. The response curve of the nominal system (Figure 9(a)) indicates that the nominal system has sufficient damping and the steady-state error is 0 . The system also retains robustness for parameter perturbation after $\mathrm{H}_{\infty}$ loop-shaping design. After related verification, the response curve with perturbation begins to diverge when the frequency of the flexible mode perturbs from $1.539 \mathrm{rad} / \mathrm{s}$ to $1.7 \mathrm{rad} / \mathrm{s}$, as shown in Figure 9(b).

In the general $\mathrm{H}_{\infty}$ theory, the system will retain stability as long as the perturbation is less than $21.07 \%$ after design according to small gain theorem. However, the system begins to diverge in Figure 9(b) when the frequency only perturbs $14 \%$, which contradicts small gain theorem. On the one hand, this is mainly because the weakly damped resonance mode will increase the norm of coprime factor perturbation, so that the allowable perturbation range is compressed. On the other hand, the $\mathrm{H}_{\infty}$ loop-shaping method is not a design aiming at robustness for a given perturbation range. It is not known how much perturbation is allowed until the design is completed. Therefore, the robustness in $\mathrm{H}_{\infty}$ loop-shaping method has its own particularity, which is different from the conventional concept.

\section{Conclusions}

We establish the mathematical model of the solar panel system in this paper and deepen the understanding of the control design for the weakly damped flexible system. And then we use $\mathrm{H}_{\infty}$ loop-shaping method combined with proper weighting function selection so as to design the stable controller for a flexible solar panel system. We try to improve system performance (bandwidth) based on the stable controller. Finally, we seek the optimal compromise between robust stability and system bandwidth. Simulation results indicate that the system after the design has sufficient robustness while maximizing bandwidth.

\section{Data Availability}

The data used to support the findings of this study are available from the corresponding author upon request.

\section{Conflicts of Interest}

The authors declare that there are no conflicts of interest regarding the publication of this paper.

\section{Acknowledgments}

This work was partly supported by the Fundamental Research Funds for the Central Universities (no. N182304010), the Natural Science Foundation of Liaoning Province (no. 20170520333), the Natural Science Foundation of Hebei Province (no. F2019501012), and the Guizhou Provincial Science and Technology Fund (no. [2020]1Y273).

\section{References}

[1] Y. V. Pisanko and O. I. Yakovlev, "Plasma polarization electric field derived from radio sounding of solar wind acceleration region with spacecraft signals," Advances in Space Research, vol. 65, no. 8, pp. 1048-1053, 2020.

[2] F. K. Arabul, A. Y. Arabul, C. F. Kumru, and A. R. Boynuegri, "“Providing energy management of a fuel cell-battery-wind turbine-solar panel hybrid off grid smart home system," International Journal of Hydrogen Energy, vol. 42, no. 43, pp. 26906-26913, 2017. 
[3] X. Liu, Y. Lei, and Q. Tang, "The attitude control of spacecraft with large flexible solar array," Journal of National University of Defense Technology, vol. 25, no. 5, pp. 6-8, 2003.

[4] Q. Chen, S. Xie, M. Sun, and X. He, "Adaptive nonsingular fixed-time attitude stabilization of uncertain spacecraft," IEEE Transactions on Aerospace and Electronic Systems, vol. 54, no. 6, pp. 2937-2950, 2018.

[5] M. Tao, Q. Chen, X. He, and M. Sun, "Adaptive fixed-time fault-tolerant control for rigid spacecraft using a double power reaching law," International Journal of Robust and Nonlinear Control, vol. 29, no. 12, pp. 4022-4040, 2019.

[6] C. Cai, N. Ning, X. Li, and Z. Nie, "Uncertainty in control design for flexible system," Journal of Harbin University of Science and Technology, vol. 19, no. 2, pp. 45-48, 2014.

[7] H. M. N. K. Balini, C. W. Scherer, and J. Witte, "Performance enhancement for AMB systems using unstable $\mathrm{H}_{\infty}$ controllers," IEEE Transactions on Control Systems Technology, vol. 19, no. 6, pp. 1479-1492, 2011.

[8] A. Pang, Z. He, M. Zhao, G. Wang, Q. Wu, and Z. Li, "Sum of squares approach for nonlinear $\mathrm{H}_{\infty}$ control," Complexity, vol. 2018, Article ID 8325609, 7 pages, 2018.

[9] F. Meng, A. Pang, X. Dong, C. Han, and X. Sha, " $\mathrm{H}_{\infty}$ optimal performance design of an unstable plant under Bode integral constraint," Complexity, vol. 2018, Article ID 4942906, 10 pages, 2018.

[10] D. McFarlane and K. Glover, "Robust controller design using normalized coprime factor plant descriptions," in Lecture Notes in Control and Information Sciences, p. 138, SpringerVerlag, New York, NY, USA, 1990.

[11] Z. He, X. Jiang, F. Meng, and G. Wang, " $\mu$-synthesis in $\mathrm{H}_{\infty}$ loop-shaping design," Control Theory \& Applications, vol. 29, no. 3, pp. 347-352, 2012.

[12] F. Meng, Z. He, G. Wang, and D. Zhou, "Control design of flexible systems and $\mathrm{H}_{\infty}$ loop-shaping method," Control Theory \& Applications, vol. 30, no. 8, pp. 1014-1020, 2013.

[13] J. Nie, R. Conway, and R. Horowitz, "Optimal $\mathrm{H}_{\infty}$ control for linear periodically time-varying systems in hard disk drives," IEEE/ASME Transactions on Mechatronics, vol. 18, no. 1, pp. 212-220, 2013.

[14] S. Wang, J. Na, and Y. Xing, "Adaptive optimal parameter estimation and control of servo mechanisms: theory and experiment," IEEE Transactions on Industrial Electronics, 2020.

[15] S. Wang and J. Na, "Parameter estimation and adaptive control for servo mechanisms with friction compensation," IEEE Transactions on Industrial Informatics, 2020.

[16] S. Wang, L. Tao, Q. Chen, J. Na, and X. Ren, "USDE-based sliding mode control for servo mechanisms with unknown system dynamics," IEEE/ASME Transactions on Mechatronics, vol. 25, no. 2, pp. 1056-1066, 2020.

[17] B. A. Francis, A Course in $H_{\infty}$ Control Theory, SpringerVerlag, New York, NY, USA, 1987.

[18] A. Lanzon and P. Tsiotras, "A combined application of $\mathrm{H}_{\infty}$ loop shaping and $\mu$-synthesis to control high-speed fly wheels," IEEE Transactions on Control Systems Technology, vol. 13, no. 5, pp. 767-777, 2005.

[19] Z. He, F.-W. Meng, W. Liu, and G.-X. Wang, "Robustness of $\mathrm{H}_{\infty}$ loop shaping design," Acta Automatic Sinica, vol. 36, no. 6 , pp. 890-893, 2010. 